\title{
Skew-symmetric tridiagonal Bohemian matrices
}

\author{
ROBERT M. CORLESS, Western University, Canada
}

\begin{abstract}
Skew-symmetric tridiagonal Bohemian matrices with population $P=[1, i]$ have eigenvalues with some interesting properties. We explore some of these here, and I prove a theorem showing that the only possible dimensions where nilpotent matrices can occur are one less than a power of two. I explicitly give a set of nilpotent matrices in this family at dimension $m=2^{k}-1$, which for $k>1$ are recursively constructed from those at smaller dimension. I conjecture that these are the only matrices in this family which are nilpotent.

This paper will chiefly be of interest to those readers of my prior paper on Bohemian matrices with this structure who want more mathematical details than was provided there, and who want details of what has been proved versus what has been conjectured by experiment.

I also give a terrible pun. Don't say you weren't warned.
\end{abstract}

CCS Concepts: • Computing methodologies $\rightarrow$ Hybrid symbolic-numeric methods.

Additional Key Words and Phrases: Bohemian matrices, nilpotent matrices, Chebyshev polynomials

\section{Recommended Reference Format:}

Robert M. Corless. 2021. Skew-symmetric tridiagonal Bohemian matrices . Maple Trans. 1, 2, Article 14360 (November 2021), 12 pages. https://doi.org/10.5206/mt.v1i2.14360

\section{Introduction and Notation}

This paper, which follows on from [5], is concerned only with skew-symmetric tridiagonal matrices with a population $P=[1, i]$ where $i$ is the square root of minus 1 ; thus the matrices are from a structured Bohemian family. A matrix family is Bohemian if its entries are from a discrete finite set; the name comes from BOunded HEight Matrices of Integers (BOHEMI). See bohemianmatrices.com for other examples. Skew-symmetric matrices have $A^{T}=-A$. Note that $A^{T}$ is not the Hermitian transpose of $A$; this is just the simple transpose without taking the complex conjugate. Tridiagonal matrices only have (at most) three nonzero bands. The diagonal entries of skew-symmetric matrices must be zero. Therefore, a skew-symmetric tridiagonal Bohemian matrix only has one diagonal of free entries, each of which must be a member of the population $P$. Here is a generic $m$-by- $m$ version of such a matrix:

$$
\mathrm{T}_{m}\left(u_{1}, u_{2}, \ldots, u_{m-1}\right)=\left[\begin{array}{cccccc}
0 & u_{1} & 0 & 0 & 0 & 0 \\
-u_{1} & 0 & u_{2} & 0 & 0 & 0 \\
0 & -u_{2} & 0 & u_{3} & 0 & 0 \\
0 & 0 & \ddots & \ddots & \ddots & 0 \\
0 & 0 & 0 & -u_{m-2} & 0 & u_{m-1} \\
0 & 0 & 0 & 0 & -u_{m-1} & 0
\end{array}\right] .
$$

Here each $u_{i}$ is a member of the population $P$. Denoting the cardinality of the population set $P$ by $\# P$, we see that the number of skew-symmetric tridiagonal matrices of dimension $m$ is $\# P^{m-1}$; in the family studied in this paper $P=[1, i]$ so $\# P=2$ and the number of matrices of dimension $m$ in

Author’s address: Robert M. Corless, rcorless@uwo.ca, Western University, London, Ontario, Canada, N6A 5B7.

Permission to make digital or hard copies of all or part of this work for any use is granted without fee, provided that copies bear this notice and the full citation on the first page. Copyrights for third-party components of this work must be honored (C) 2021 Copyright held by the owner/author(s). Publication rights licensed to Maple Transactions, under Creative Commons CC-BY 4.0 License.

https://doi.org/10.5206/mt.v1i2.14360

Maple Trans., Vol. 1, No. 2, Article 14360. Publication date: November 2021. 
this family is just $2^{m-1}$. This is actually a rather small Bohemian family; many families have size $\# P^{O\left(m^{2}\right)}$, which can be vastly larger ${ }^{1}$.

Why on earth study this Bohemian family? I arrived at this family by simplifying a related family, complex symmetric tridiagonal Bohemians, which were interesting for other reasons (every matrix is similar to a complex symmetric matrix); making the diagonal entries all zero simplified the family quite a lot without grossly losing information (the average of the population I was looking at then was zero, and so replacing the diagonal with zero just made the eigenvalue diagrams skinnier) and then rotating in the complex plane made skew-symmetry natural. Then the intrinsic properties of the family made themselves known; there are surprises here. See [5] for details.

The height of a matrix is the maximum absolute value of any entry in the matrix; in other words, the vector infinity norm of the matrix when considered as a vector (either by stacking the columns into a single column vector, or by appending the rows into a single row vector): $\operatorname{height}(\mathrm{T})=\|\operatorname{vec}(\mathrm{T})\|_{\infty}$.

We write $p_{m}\left(\lambda ; u_{1}, \ldots, u_{m-1}\right)$ for a polynomial in $\lambda$ of degree $m$ that depends on the parameters $u_{1}, u_{2}, \ldots, u_{m-1}$. The characteristic height of a polynomial $p$ is the maximum absolute value of any coefficient; in other words, the infinity norm of the vector of coefficients. We use this term even for generic polynomials (where we could just get away with calling it the "height") as opposed to for polynomials that arise as the characteristic polynomial of a matrix, where there might be confusion between the height of a polynomial and the height of a matrix it comes from. By using "characteristic height" for a polynomial, we keep the notions separate and less confusing.

$$
\text { characteristic height }(p)=\max _{0 \leq k \leq m}\left|\left[\lambda^{k}\right] p\right| .
$$

Here the notation $\left[\lambda^{k}\right] p$ means the coefficient $a_{k}$ in $p(\lambda)=a_{0}+a_{1} \lambda+\cdots+a_{m} \lambda^{m}$. See [6, p. 197] and [9] for more discussion of this notation.

When there is no ambiguity as to the value of the parameters, we will write $\mathbf{T}_{m}$ and $p_{m}$ or perhaps $p_{m}(\lambda)$, leaving the parameters implicit.

\subsection{Overview of this paper}

In section 2 we sketch some lemmas and propositions we need in order to motivate and prove Theorem 4.1, which definitively answers the question of when matrices in this family can be nilpotent.

We remind you that the reason this is interesting is that the visibility of gross rounding errors in the numerical computation of eigenvalues of all matrices in this family of dimensions $m=7$ and $m=15$ (and later confirmation at $m=3$ ) led to this conjecture; normally, eigenvalue computation of such small dimension matrices is extremely reliable, typically much more so than numerical rootfinding of polynomials expressed in the monomial basis. See [4] for a discussion of why this rule is generally true. Indeed, according to proposition 2.12., eigenvalue computation is generally more reliable than polynomial rootfinding is for this family, as well. So, the visible rounding errors in the pictures in [5] needed an explanation. Theorem 4.1 shows that the unexpected behaviour can only happen at dimension one less than a power of two, and the combinatorial proof, which depends on some classical results, was surprising to me (and kind of fun).

Next, in Theorem 4.2, I give a recursive construction for nilpotent matrices in this family at each dimension $m=2^{k}-1$ for $k>1$. This shows that there really are a few $\left(2^{k-1}\right)$ nilpotent matrices at dimension $2^{k}-1$; given that there are $2^{2^{k}-2}$ matrices in this family at that dimension, encountering such matrices "at random" is not very likely!

${ }^{1}$ It might be surprising to realize just how much faster $2^{m^{2}}$ grows than $m$ ! does. Try it! 
In Conjecture 5.1 I conjecture that these are the only nilpotent matrices in this family. I'm not all that confident that this is true, although I have checked up to $m=31$ (at that dimension, there are more than a billion matrices; I checked by computing the characteristic polynomials by the recursion relation (3), in Julia). I'd be quite interested in a proof.

I end by suggesting some further reading. There is a lot of activity in this area; some classical, and some where we barely know even what the easy questions are.

\section{First steps}

We begin by proving (or sketching proofs of) the following propositions. Some of them are true for general populations $P$. This first proposition was left as an exercise in [5] and we only sketch the proof here.

Proposition 2.1. The characteristic polynomial $p_{m}\left(\lambda ; u_{1}, u_{2}, \ldots, u_{m-1}\right)$ of $\mathbf{T}_{m}\left(u_{1}, u_{2}, \ldots, u_{m-1}\right)$ satisfies the recurrence relation

$$
p_{m+1}\left(\lambda ; u_{1}, u_{2}, \ldots, u_{m}\right)=\lambda p_{m}\left(\lambda ; u_{1}, u_{2}, \ldots, u_{m-1}\right)+u_{m}^{2} p_{m-1}\left(\lambda ; u_{1}, u_{2}, \ldots, u_{m-2}\right),
$$

with $p_{0}(\lambda ; \varnothing)=1$ by convention, and $p_{1}(\lambda ; \varnothing)=\lambda$.

Proof. We make $\mathbf{T}_{0}$ the empty matrix and simply declare its characteristic polynomial to be 1 . The only skew-symmetric matrix of dimension $m=1$ is $\mathbf{T}_{1}=[0]$ which has characteristic polynomial $p_{1}(\lambda)=\lambda$. Direct computation of $\operatorname{det}\left(\lambda \mathbf{I}-\mathrm{T}_{2}\right)$ gives $\lambda^{2}+u_{1}^{2}$, which retroactively ensures that the base of the induction agrees with the convention that $p_{0}=1$. Choose a positive integer $M$ and suppose the theorem is true for all $m+1<M$. Consider the $M$-by- $M$ matrix $\mathbf{T}_{M}$. Laplace expansion about the $(M, M)$ corner element gives us, in two steps, the desired recurrence relation.

This already tells us quite a bit about the eigenvalues of any $\mathbf{T}_{m}$ when the population is $P=[1, i]$. We have the following propositions:

2.2. If $m$ is odd, then there is a polynomial $q(z)$ of degree $\lfloor m / 2\rfloor$ with $p_{m}\left(\lambda ; u_{1}, u_{2}, \ldots, u_{m-1}\right)=$ $\lambda q\left(\lambda^{2}\right)$. Thus if $m$ is odd, $\mathbf{T}_{m}$ is singular.

2.3. If $m$ is even, then there is a polynomial $q(z)$ of degree $m / 2$ with $p_{m}\left(\lambda ; u_{1}, u_{2}, \ldots, u_{m-1}\right)=q\left(\lambda^{2}\right)$. Thus if $m$ is even and no $u_{j}$ is zero ${ }^{2}, \mathbf{T}_{m}$ is nonsingular.

2.4. If $z$ is an eigenvalue of $\mathbf{T}_{m}\left(u_{1}, u_{2}, \ldots, u_{m-1}\right)$, then so is $-z$.

2.5. If $z$ is an eigenvalue of $T_{m}\left(u_{1}, u_{2}, \ldots, u_{m-1}\right)$ then $z$ is an eigenvalue of $T\left( \pm u_{1}, \pm u_{2}, \ldots, \pm u_{m}\right)$ because only $u_{k}^{2}$ enters the characteristic polynomial.

2.6. If $i$ is in the population $P$, then eigenvalues might be complex. If the population is such that all $u_{j}^{2}$ are real, then the coefficients of the characteristic polynomials are real; hence if complex $z$ is an eigenvalue, so is $\bar{z}$.

Remark: The resulting four-fold symmetry of the set of eigenvalues of all matrices $\mathbf{T}_{m}$ (for fixed $m$, as the parameters range over all $2^{m-1}$ possible sets of parameters) when $P=[1, i]$ was already used in [5] to determine that the visible "rose" in the computed spectra near $\lambda=0$ in the case $m=7$ must have been due to rounding errors, because it exhibited seven-fold symmetry, which is impossible because odd symmetries are not compatible with the four-fold symmetry.

2.7. Fix a sequence $u_{1}, u_{2}, \ldots$ of elements of the population $P$, with no $u_{i}=0$ (our population $P$ does not contain zero, but this fact holds for other populations as well). Then the gcd of $p_{i}\left(\lambda ; u_{1}, \ldots, u_{i-1}\right)$ and $p_{i+1}\left(\lambda ; u_{1}, \ldots, u_{i}\right)$ is 1 .

\footnotetext{
${ }^{2}$ To see that we need the $u_{j}$ to be nonzero, look at the recurrence relation; if any $u_{j}$ is zero, then $T_{j+1}$ is zero at $\lambda=0$ and thereafter any $T_{k}$ with $k>j$ will also have a zero at $\lambda=0$.
} 
Proof. The proof is by induction and contradiction: since $p_{0}=1$ and $p_{1}=\lambda$ have no common roots, and if any pair of consecutive polynomials had a common root it must also be a root of $p_{i-1}\left(\lambda ; u_{1}, \ldots, u_{i-2}\right)$ by the recurrence relation in equation (3). Induction downwards gives the contradiction.

2.8. For population $P=[1, i]$, the maximal characteristic height $H_{m}$ of any $\mathbf{T}_{m}$ occurs exactly twice: when all $u_{k}=1$ and when all $u_{k}=i$.

Proof. Again the proof is by induction. When $m=0$ there is only $p=1$. When $m=1$ there is only $p=\lambda$. When $m=2$ there are two polynomials, of equal and maximal height: call them $r_{2}=\lambda+1$ and $s_{2}=\lambda-1$. When $m=3$ there could be four polynomials, but two of them are the same, so there are only three distinct polynomials, $r_{3}=\lambda^{3}+2 \lambda$, $p_{2}=\lambda^{3}$, and $s_{3}=\lambda_{3}-2 \lambda$. Let us start the induction at $m=3$. Now suppose that at $m=k$ and at $m=k-1$ there are only two polynomials, for each $m$, of maximal height: $r_{k}$ and $r_{k-1}$ with positive coefficients and $s_{k}$ and $s_{k-1}$ with alternating coefficients. Now consider any $p_{k+1}=\lambda p_{k}+u_{k}^{2} p_{k-1}$. Define

$$
\begin{aligned}
& r_{k+1}=\lambda r_{k}+r_{k-1} \\
& s_{k+1}=\lambda s_{k}-s_{k-1} .
\end{aligned}
$$

The first can happen by choosing $u_{k}=1$, and the second by choosing $u_{k}=i$. Quite obviously, no $\lambda p_{k}+p_{k-1}$ can have a larger coefficient than $r_{k+1}$ can; it is not quite so obvious but $s_{k+1}$ has the same height (indeed the same coefficients), with alternating signs. That is, $s_{m}(\lambda)=i^{m} r_{m}(-i \lambda)$. Moreover, choosing any strictly lower height $p_{k}$ or $p_{k-1}$ must lead to a strictly lower height $p_{k+1}$.

2.9. $\left[\lambda^{k}\right] r_{m}(\lambda)$ counts the number of terms in $\left[\lambda^{k}\right] P_{m}\left(\lambda ; u_{1}, u_{2}, \ldots, u_{m-1}\right)$ with symbolic $u_{j}$. For example, $P_{6}(\lambda)=\lambda^{6}+\left(u_{1}^{2}+u_{2}^{2}+u_{3}^{2}+u_{4}^{2}+u_{5}^{2}\right) \lambda^{4}+\left(u_{1}^{2} u_{3}^{2}+u_{1}^{2} u_{4}^{2}+u_{1}^{2} u_{5}^{2}+u_{2}^{2} u_{4}^{2}+u_{2}^{2} u_{5}^{2}+u_{3}^{2} u_{5}^{2}\right) \lambda^{2}+$ $u_{1}^{2} u_{3}^{2} u_{5}^{2}$ and $r_{6}(\lambda)=\lambda^{6}+5 \lambda^{4}+6 \lambda^{2}+1$. We see directly that there is a sum of five terms in the coefficient of $\lambda^{4}$, a sum of six terms in the coefficient of $\lambda^{2}$, and only one term (a product) in the constant coefficient. This counting proposition can be seen by observing that $u_{m}^{2}$ occurs only once in any iteration of the recurrence, and the product involving it gets added to every nonzero term.

2.10. $r_{m}(\lambda)=F_{m+1}(\lambda)$ is the $m+1$ st Fibonacci polynomial.

2.11. $s_{m}(\lambda)=U_{m}(\lambda / 2)=i^{m} F_{m+1}(-i \lambda)$ where $U_{m}(z)$ is the $m$ th Chebyshev polynomial of the second kind. These polynomials have explicitly known coefficients:

$$
\begin{aligned}
U_{m}(z) & =(m+1) F\left(\begin{array}{c}
m+2,-m \mid \frac{1-z}{2} \\
3 / 2
\end{array}\right) \\
& =(m+1) \sum_{k \geq 0} \frac{(m+2)^{\bar{k}}(-m)^{\bar{k}}}{(3 / 2)^{\bar{k}}} \frac{((1-z) / 2)^{k}}{k !} \\
& =\sum_{k=0}^{\lfloor m / 2\rfloor}(-1)^{k}\left(\begin{array}{c}
m-k \\
k
\end{array}\right)(2 z)^{m-2 k} .
\end{aligned}
$$

This connection between Fibonacci polynomials and Chebyshev polynomials of the second kind is well-known; indeed Bill Gosper likes to say that $\left|U_{m}(i / 2)\right|$ is the "shortest formula for the Fibonacci numbers".

2.12. By using the fact that the length of a polynomial, i.e. the 1-norm of the vector of coefficients, is bigger than the height of a polynomial, one can see that

$$
\frac{1}{m+1} F_{m+1}<H_{m}<F_{m+1}
$$


where $F_{0}=0, F_{1}=1$, and $F_{k+1}=F_{k}+F_{k-1}$ are the Fibonacci numbers. This makes the claimed asymptotic estimate in the following item at least plausible. More, put $f(k ; m)=\left(\begin{array}{c}m-k \\ k\end{array}\right)$ and consider $f(k+1 ; m)-f(k ; m)$. Setting this to zero leads to $k=m / 2-3 / 10-\sqrt{5 m^{2}+10 m+9} / 10$. This is not generally an integer but will be close to where $f(k ; m)$ is maximum. Putting this value of $k$ into $f(k ; m)$, using asympt and simplifying ${ }^{3}$ confirms the following asymptotics.

2.13. The sequence of heights $H_{m}$ of these polynomials (the maximal characteristic heights of the matrices) is given by http://oeis.org/A073028, where we see the claim that the asymptotics as $m \rightarrow \infty$ is given by

$$
H_{m} \sim A_{m}:=\frac{5^{1 / 4}}{\sqrt{2 \pi(m+1)}} \phi^{m+1}
$$

where $\phi=(1+\sqrt{5}) / 2$ is the golden ratio.

A very similar bound and asymptotic result (actually, conjecture) appear in [3]; see Theorem 4.20 and Figure 1 of that paper in particular; we have a very similar figure here, Figure 1(a), which shows experimentally that

$$
-\frac{1.4}{m}<\frac{H_{m}-A_{m}}{H_{m}}<\frac{0.2}{m}
$$

over quite a wide range of $m$. This suggests that the relative error in approximating $H_{m}$ by using $A_{m}$ decays like $O(1 / m)$. The detailed error behaviour, however, is quite interestingly complicated, and similar to that in [3].

There is a reason for that, of course. Both figures show the error in approximating the largest binomial coefficient with a formula that comes from a continuous approximation. The reason that they resemble the "Thousand Points of Light" from [8], however, is because the binomial coefficient is an integer; the maximum coefficient does not occur where the real-valued formula predicts it should, but rather at a truncation of that. This introduces a $\sin 2 \pi c n$ like term into the error formula (here $c$ is some algebraic constant). See Appendix A. In figure 1(b), we see qualitatively similar behaviour by plotting $\sin 2 \pi c n$ against (integer) $n$ on the range $1000 \leq n \leq 5000$, with $c=1 / T$ where $T$ is an estimate of the period of this fractional part error. The following Maple commands were used to produce Figure 1(b). See [10] for a nice explanation of what is going on, in physics terms.

$$
\begin{gathered}
>\operatorname{plot}\left(\left[\operatorname{seq}\left(\left[n, \sin \left(\frac{2 \cdot \mathrm{Pi} \cdot 2 \cdot n}{5+\operatorname{sqrt}(5)}\right)\right], n=1000 . .5000\right)\right], \text { style }=\right.\text { point, symbol = point, colour = black, } \\
\text { axes } \left.=\text { boxed, labels }=\left[n, \text { typeset }\left(\sin \left(\frac{2 \cdot 2 \cdot \mathrm{Pi} \cdot n}{5+\operatorname{sqr}(5)}\right)\right)\right], \text { labeldirections }=[\text { horizontal, vertical }]\right)
\end{gathered}
$$

Remark This is quite the result. We have proved that the height of the characteristic polynomial must grow exponentially with $n$, while the height of the matrix, which is just 1 , remains bounded. This has a numerical consequence: the roots of the characteristic polynomials must have an exponentiallygrowing condition number (recall that the condition number of a polynomial expressed in the monomial basis as $p(x)=\sum a_{j} x^{j}$ is $B(x)=\sum\left|a_{j}\right||x|^{j}$ and so exponentially-growing $a_{j}$ means exponentially growing $B(x)$, even if the values of $p(x)$ are small), and thus require a linearly-growing number of Digits in order to solve the polynomials accurately. In contrast, the eigenvalues of the matrix can be computed in ordinary hardware precision until the dimension is so large that its square is about the reciprocal of machine epsilon; in double precision, this means until the dimension is about $m=10^{8}$. Computation will be expensive for such a large dimension, because one

\footnotetext{
${ }^{3}$ Normally, I would include the Maple commands to do this here. However, I had a hard time finding a neat way to do so and I had to ask for help via Maple Primes. Acer was able to answer my question and showed two good tricks to do this, and so I recommend that you look at his answer at that link.
} 


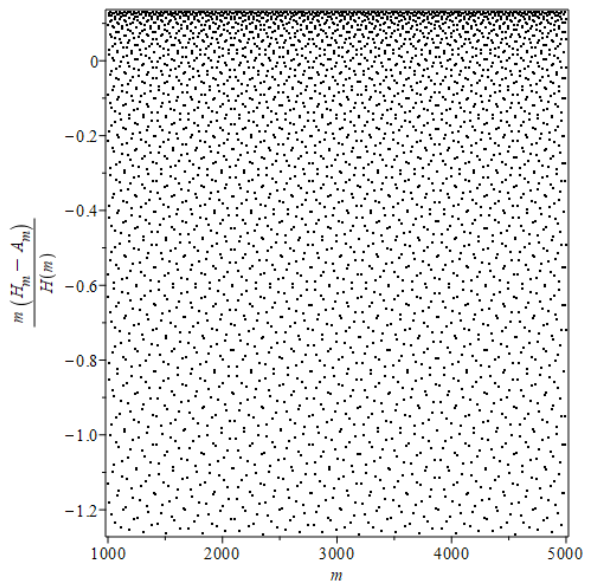

(a) Relative Error in Asymptotic Formula

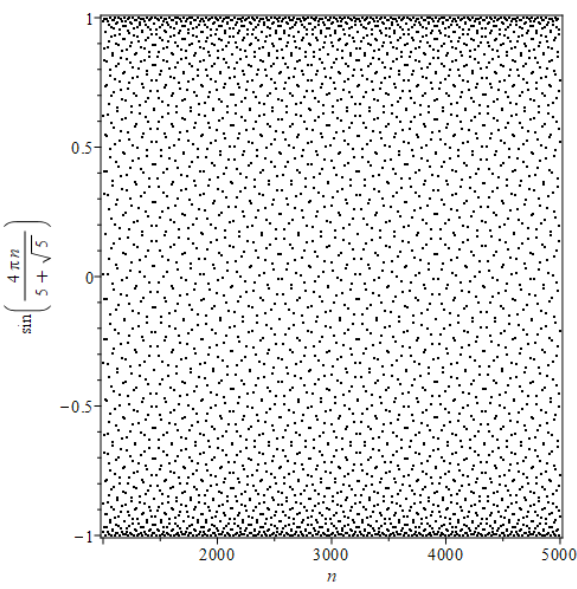

(b) Sampling sine at discrete points

Fig. 1. The plot on the left shows the relative error $-1.4 / m<\left(H_{m}-A_{m}\right) / H_{m}<0.2 / m$ in the asymptotic formula over the range $1000 \leq m \leq 5000$. Compare with Figure 1 of [3], which has a remarkable resemblance. The plot on the right shows a sampling the sine function at integer multiples of an algebraic number times $2 \pi$, which produces a very similar pattern. Using a different algebraic number produces visibly different patterns. Here the algebraic number is $1 / T=2 /(5+\sqrt{5})$, where $T$ is (asymptotically) the period of the fractional error The vertical scale is not accounted for in this analysis, which is only qualitative (but very remarkable). See Appendix A. See also [8] for an early investigation of this phenomenon, and [10] for what seems to be the right general explanation.

will be computing about a hundred million eigenvalues, but because the matrix is only tridiagonal there exist special algorithms whose cost only grows linearly with the dimension; this is something that can actually be accomplished with today's computers. In contrast, even using the fastest available polynomial solvers on the characteristic polynomial is much slower, and in the same time we could only achieve a much lower dimension $m$.

\section{Even so, eigenvalue computation can have problems too}

The whole reason that we care about nilpotent matrices was explored in [5]: namely, computing the eigenvalues of nilpotent matrices will, owing to rounding errors, produce spuriously nonzero numbers about the size of $\mu^{1 / m}$, where $\mu=2^{-53} \approx 10^{-16}$ is the unit roundoff. If $m=31$ then $\mu^{1 / m} \approx 0.3$ and there's no wonder that the rounding errors are visible. More startling is the symmetry, but that is explained partially by the solution of the perturbed system $z^{m}-\mu=0$, which has its roots scattered fairly uniformly around the cirle of approximate radius 0.3 . In this case it is by far better to generate the explicit polynomial, $z^{m}=0$, and solve that! One therefore needs both numerical and symbolic computation for this problem.

\section{Formal results}

Theorem 4.1. If $\mathrm{T}_{m}$ is nilpotent, then $m=2^{k}-1$ for some $k$.

Proof. We use the "counting" proposition 2.9. and the known values $\left(\begin{array}{c}m-k \\ k\end{array}\right)$ for the coefficients of $r_{m}(\lambda)$. Each term in the sums making up the coefficients of $p_{m}\left(\lambda ; u_{1}, \ldots, u_{m-1}\right)$ must be either 1 or -1 . A necessary condition for these to be able to sum to zero is that the number of terms be even; 


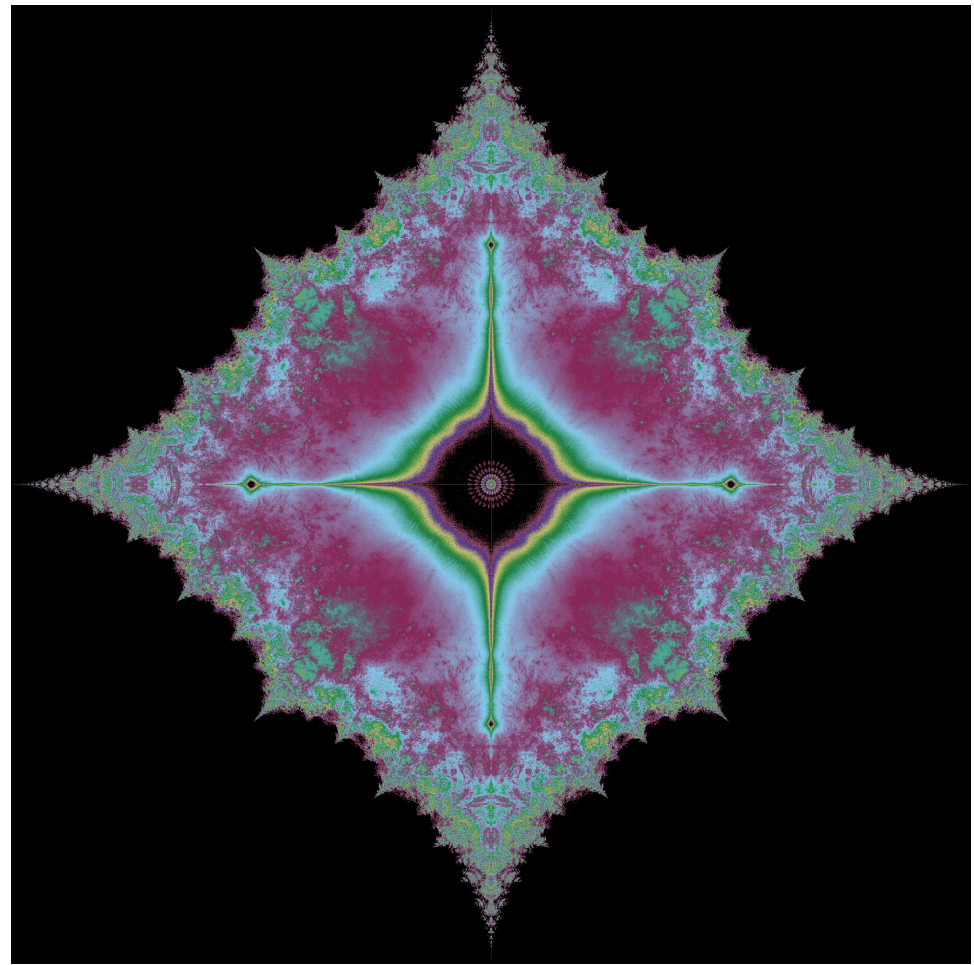

Fig. 2. Eigenvalues of all 1,073, 741, 824 skew-symmetric tridiagonal Bohemian matrices of dimension $m=31$ with population $[1, i]$. Computation took 85 hours using double precision on a 32 core machine ("scg" at the University of Waterloo). Compiled C program and artistic choices for colours by Aaron Asner. The rosette in the middle is entirely due to rounding errors, owing to the 32 nilpotent matrices in this enormous family.

if on the contrary there are an odd number of unit magnitude terms, then the sum cannot be zero. Thus we need to know the values of $m$ for which the binomial coefficients $\left(\begin{array}{c}m-k \\ k\end{array}\right)$ for $1 \leq k \leq\lfloor m / 2\rfloor$ are all even. We use Lucas's theorem [18] (a classical theorem about prime divisors in binomial coefficients; see the prize-winning paper [7] for more fun along these lines) to demonstrate that unless $m$ is one less than a power of two, at least one $\left(\begin{array}{c}m-k \\ k\end{array}\right)$ is odd for $1 \leq k \leq\lfloor m / 2\rfloor$. In fact we use a corollary to Lucas's theorem, namely that $\left(\begin{array}{c}m-k \\ k\end{array}\right)$ will be divisible by 2 if and only if at least one of the bits of $k$ is greater than the corresponding bit of $m-k$.

We proceed by contradiction. Suppose $m$ has a zero bit somewhere in it, after the highest bit which must be 1 . Let $j$, counting from zero, indicate the position of the first 0 bit in $m$. Now take $k=2^{j}$ and compare the bits in $m-k$ to the bits in $k$. Subtracting $2^{j}$ from $m$ necessarily produces a 1 bit in the $j$ th position of $m-k$ (borrowing from some higher bit, which must exist by hypothesis) and does not change any lower-index bit of $m$, so they remain all 1s. Then $k$ has no bit greater than any corresponding one in $m-k$ and thus, by the corollary to Lucas's theorem, $\left(\begin{array}{c}m-k \\ k\end{array}\right)$ is odd.

Therefore for all $\left(\begin{array}{c}m-k \\ k\end{array}\right)$ to be even, it is necessary that all bits of $m$ must be 1 , and therefore $m=2^{k}-1$ for some $k$.

Theorem 4.2. Let $n=2^{k}-1$ and $m=2^{k-1}-1$, so $n=2 m+1$. If $\mathbf{u}_{m}=\left[u_{1}, u_{2}, \ldots, u_{m-1}\right]$, and if $\operatorname{rev}\left(\mathbf{u}_{m}\right)=\left[u_{m-1}, \ldots, u_{2}, u_{1}\right]$ is that vector reversed, and if $\mathbf{u}_{n}=\left[\mathbf{u}_{m}, 1, i, \operatorname{rev}\left(\mathbf{u}_{m}\right)\right]$ or if $\mathbf{u}_{n}=$ 
$\left[\mathbf{u}_{m}, i, 1, \operatorname{rev}\left(\mathbf{u}_{m}\right)\right]$ and further if $\mathbf{T}_{m}\left(\mathbf{u}_{m}\right)$ is nilpotent, then so is $\mathbf{T}_{n}\left(\mathbf{u}_{n}\right)$. That is, we can recursively build two vectors that will each give a nilpotent matrix of dimension $n=2^{k}-1$ out of every smaller vector that makes a nilpotent matrix of dimension $m=2^{k-1}-1$.

Proof. The proof is not technically difficult, requiring only Laplace expansion of $\mathbf{R}_{n}(\lambda)=\lambda \mathbf{I}-\mathbf{T}_{n}$ about the central diagonal entry $R_{m+1, m+1}$. But it requires some care with indices. To make it a little bit simpler, let $\sigma$ be one of $\{1, i\}$ and $\tau$ be the other one, so $\sigma^{2}+\tau^{2}=0$. Then we can handle both cases at once. Then we can partition $\mathbf{R}_{n}$ as

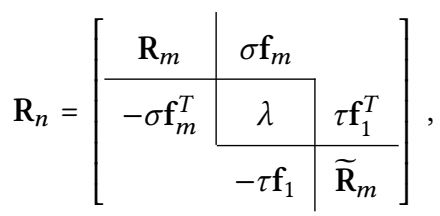

where $\mathbf{f}_{j}$ is the $j$ th elementary vector of dimension $m$. We further partition the smaller $\mathbf{R}_{m}$ into

$$
\mathbf{R}_{m}=\left[\begin{array}{c|c}
\mathbf{V}_{m-1} & u_{m-1} \mathbf{e}_{m-1} \\
\hline-u_{m-1} \mathbf{e}_{m-1}^{T} & \lambda
\end{array}\right]
$$

where $\mathbf{e}_{k}$ is now the $k$ th elementary vector of dimension $m-1$. Partition its reversal $\widetilde{\mathbf{R}}_{m}=\mathbf{S}_{m} \mathbf{R}_{m} \mathbf{S}_{m}$ in an analogous fashion, with

$$
\widetilde{\mathbf{V}}_{m-1}=\mathrm{S}_{m-1} \mathbf{V}_{m-1} \mathrm{~S}_{m-1}
$$

where $\mathrm{S}_{r}$ is the $r$-dimensional standard involutory permutation (SIP), with ones on the antidiagonal and zeros elsewhere. This matrix is also called the anti-identity. We have $\mathrm{S}_{k}^{2}=\mathbf{I}_{k}$ and $\operatorname{det} \mathrm{S}_{k}=(-1)^{k-1}$ so the SIP transformation $\tilde{\mathbf{R}}=$ SRS preserves the determinant (indeed, the characteristic polynomial).

Now $\operatorname{det} \widetilde{\mathbf{R}}_{m}=\operatorname{det} \mathbf{R}_{m}$ and by hypothesis $\operatorname{det} \mathbf{R}_{m}=\lambda^{m}$. Laplace expansion about the central row (or central column) of $\mathbf{R}_{n}$ gives

$$
\operatorname{det} \mathbf{R}_{n}=\lambda M_{1}-\sigma M_{2}+\tau M_{3}
$$

where the minors are

$$
\begin{aligned}
& M_{1}=\operatorname{det}\left[\begin{array}{l|l}
\mathbf{R}_{m} & \\
\hline & \widetilde{\mathbf{R}}_{m}
\end{array}\right], \\
& M_{2}=\operatorname{det}\left[\begin{array}{c|c|cc}
\mathbf{V}_{m-1} & u_{m-1} \mathbf{e}_{m-1} & & \\
\hline & -\sigma & \tau & \\
& & \lambda & u_{m-1} \mathbf{e}_{1}^{T} \\
& & -u_{m-1} \mathbf{e}_{1} & \widetilde{\mathbf{V}}_{m-1}
\end{array}\right] \text {, }
\end{aligned}
$$

and

$$
M_{3}=\operatorname{det}\left[\begin{array}{cc|c|c}
\mathbf{V}_{m-1} & u_{m-1} \mathbf{e}_{m-1} & \multicolumn{2}{|l}{} \\
-u_{m-1} \mathbf{e}_{m-1}^{T} & \lambda & \multicolumn{2}{|l}{} \\
\hline & -\sigma & \tau & \\
& & -u_{m-1} \mathbf{e}_{1} & \widetilde{\mathbf{V}}_{m-1}
\end{array}\right]
$$


These minors are all block diagonal, so their determinants are straightforward. This gives

$$
\begin{aligned}
\operatorname{det} \mathbf{R}_{n}= & \lambda \operatorname{det} \mathbf{R}_{m} \operatorname{det} \widetilde{\mathbf{R}}_{m}+\sigma^{2} \operatorname{det} \mathbf{V}_{m-1} \operatorname{det} \widetilde{\mathbf{R}}_{m} \\
& +\tau^{2} \operatorname{det} \widetilde{\mathbf{V}}_{m-1} \operatorname{det} \mathbf{R}_{m} \\
= & \lambda \lambda^{m} \lambda^{m}=\lambda^{n},
\end{aligned}
$$

because det $\widetilde{\mathbf{V}}_{m-1}=\operatorname{det} \mathbf{V}_{m-1}$, det $\widetilde{\mathbf{R}}_{m}=\operatorname{det} \mathbf{R}_{m}=\lambda^{m}$, and $\sigma^{2}+\tau^{2}=0$.

\section{A conjecture}

I thought I had a proof for the following conjecture, but on closer inspection I did not. This conjecture has been experimentally verified out to $m=31$ using exhaustive computation ${ }^{4}$, but to verify it computationally for $m=63$ would require about 73,000 more years of computing, or else more intelligence. I'm working on that.

CONJECTURE 5.1. The vectors recursively constructed according to the rule given in the last theorem ${ }^{5}$ give the only nilpotent matrices in the Bohemian family of skew-symmetric tridiagonal matrices with population $P=[1, i]$.

Remark This conjecture needs proof, in spite of the computational evidence above. This is because the nature of multiple eigenvalues at 0 is not very regular. By direct computation with $m=7$, in addition to matrices with double eigenvalues, there are matrices with 3-fold eigenvalues, 5 -fold eigenvalues, and of course 7-fold eigenvalues. Again by direct computation with $m=15$ there are matrices with 3-fold, 5-fold, 7-fold, 11-fold, 13-fold, and 15-fold eigenvalues-but none with 9-fold eigenvalues. Will other irregularities arise at higher dimension?

Conjecture 5.1 is equivalent to showing that the matrix $Q_{m}$ bringing $\mathbf{T}_{m}$ to Jordan Canonical Form, i.e. $\mathbf{T}_{m} \mathbf{Q}_{m}=\mathbf{Q}_{m} \mathbf{J}_{m}$ where $\mathbf{J}_{m}$ is a full Jordan block of zeros, has the form

$$
\mathbf{Q}_{m}=\left[\begin{array}{ccc}
\mathbf{L} & 0 & \mathbf{A}_{1} \\
0 & x & \\
\mathbf{A}_{2} & &
\end{array}\right]
$$

where $x$ is scalar, $\mathbf{L}$ is lower triangular and both $\mathrm{A}_{1}$ and $\mathrm{A}_{2}$ (and, therefore, $\mathbf{Q}_{m}$ ) are what is known as upper anti-triangular. See [11] for other anti-triangular matrices in the literature. When $m=15$,

\footnotetext{
${ }^{4}$ A serial computation in Julia (actually, my very first Julia program, so possibly not as fast as it could be) on a $1.3 \mathrm{GHz}$ Surface Pro tested all $2^{30}>1.07 \cdot 10^{9}$ permutations for $m=31$ (using 64-bit integer arithmetic, which is enough because the 32nd Fibonacci number needs only 22 bits) in 542 seconds.

${ }^{5}$ Because their Jordan form is a single block with all zero eigenvalues, i.e. they have no heart, no centre, no "core", I propose to call these "coreless matrices." [I did promise (threaten?) an awful pun in [5]!]
} 
the matrix looks like this, where $x$ means a nonzero element ${ }^{6}$ :

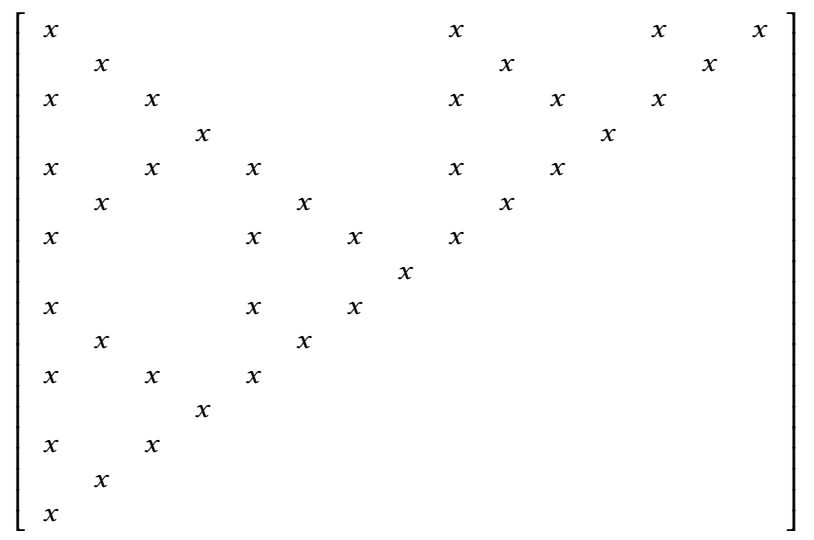

We can already see the Sierpinski gasket forming.

As a corollary, we could say then that there is one nilpotent matrix in this family of dimension $1=2^{1}-1$, two of dimension $3=2^{2}-1$, four of dimension $7=2^{3}-1$, and so on: inductively there would be only $2^{k-1}$ nilpotent matrices in this family of dimension $2^{k}-1$. Since there are $2^{2^{k}-2}$ matrices of this dimension in this family, this means that the probability of finding a nilpotent matrix by choosing "at random" becomes vanishingly small with the dimension: $\left(2^{k}-1\right) / 2^{2^{k}-2} \sim 2^{k-2^{k}}$.

\section{Further Reading}

Olga Taussky-Todd was one of the pioneers of Bohemian matrices (although she did not call them that); see [15] for her survey of the subject. Relevant papers of her own for this present work include [17], which states that general skew-symmetric matrices may be unitarily transformed to tridiagonal ones; and [16], which proves some related results about the matrices studied in this paper. That paper also proves further that zero is included in the convex hull of the eigenvalues; this is a key feature of the symmetry of the pictures in [5]. Several of her papers discuss the connection between algebraic number theory and (what I now call) Bohemian matrices, and I look forward to reading them all.

Is there a sequence more popularly recognizable than the Fibonacci numbers? Likely not, although their deep history is probably not as widely known as it should be: the sequence 1, 1, 2, 3, $5,8, \cdots$ was in fact known long before Leonardo of Pisa published his Liber Abaci, indeed at least as early as 200BCE in Pingala's study of Sanskrit prosody ${ }^{7}$ [19]. Pingala also knew binary numbers and Pascal's triangle, both of which were important for this paper.

Nowadays there are journals devoted to Fibonacci (Pingala) numbers, there are exhibits at national art galleries by artists inspired by them, and there is a lovely article debunking a lot of the hype surrounding them [13]. But, over-hyped or not, misattributed or not, they are fun, and the students find them fascinating, as well as their generalizations such as the Narayana numbers ${ }^{8}$ (see [2]).

The interest in the height of a polynomial (and its length, for that matter; the height and length are the $\infty$-norm and 1-norm, respectively, of the vector of coefficients of the polynomial) goes back at least to the work of Kurt Mahler [12]. That paper also introduces, besides the height and length

\footnotetext{
${ }^{6}$ Maple's "matrix browse" will show the structure of a matrix in a number of useful ways, but the result is hard to export. A view of the structure of a matrix can be replicated by applying map $(t->$ ifelse $(t=0, b, x), A)$, converting the result to ${ }_{\mathrm{AT}} \mathrm{TX}$, and editing the results to change all the "b"s to blank spaces. This kludge is occasionally helpful.

${ }^{7}$ According to Neil Calkin, this is Applied Mathematics: The application is the study of poetry!

${ }^{8}$ The Narayana cow's sequence satisfies the recurrence $r_{n}=r_{n-1}+r_{n-3}$ and starts with one cow.
} 
of a polynomial, a multiplicative measure now known as the Mahler measure. There are several unsolved problems and conjectures relating to the Mahler measure. Interestingly enough, that paper just cited contains a conjecture about polynomials with \pm 1 coefficients, which are nowadays known as Littlewood polynomials. According to a footnote added in proof, that conjecture was disproved by early experimental computation, on an "Iliac II" computer at the University of Illinois, by "Mr. Michael Comenatz and Mr. Stephen Grant, students in the 1963 Undergraduate Summer Program." Experimental mathematics was alive and kicking, then! The paper is still well worth reading today.

Recent work entitled The Sharp Erdős-Turán Inequality by Shu and Wang [14] shows that the original interest in these topics still survives. The theory of Bohemian matrices grew out of these studies, and out of early work by Peter Borwein and Loki Jörgenson [1]. Again, those papers are well worth reading.

\section{Supporting Material}

Supporting material for this paper can be found at this Maple Cloud link and this GitHub link.

\section{Acknowledgments}

Several people helped with this project, including Aaron Asner, Neil J. Calkin, Eunice Y.S. Chan, Jürgen Gerhard, Laureano Gonzalez-Vega, David W. Linder, Erik Postma, J. Rafael Sendra, Juana Sendra, and Steven Thornton.

This work was partially supported by the grant PID2020-113192GB-I00 (Mathematical Visualization: Foundations, Algorithms and Applications) from the Spanish MICINN, and by NSERC Grant RGPIN-2020-06438.

\section{References}

[1] Peter Borwein and Loki Jörgenson. "Visible structures in number theory". In: The American Mathematical Monthly 108.10 (2001), pp. 897-910 (cit. on p. 11).

[2] Eunice Y. S. Chan. "A comparison of solution methods for Mandelbrot-like polynomials". MA thesis. London, Canada: Western University, 2016. URL: ir.lib.uwo.ca/etd/4028 (cit. on p. 10).

[3] Eunice Y.S. Chan et al. "Upper Hessenberg and Toeplitz Bohemians". In: Linear Algebra and its Applications 601 (Sept. 2020), pp. 72-100. URL: https://doi.org/10.1016/j.laa.2020.03.037 (cit. on pp. 5, 6).

[4] Robert M Corless and Leili Rafiee Sevyeri. "The Runge Example for Interpolation and Wilkinson's Examples for Rootfinding”. In: SIAM Review 62.1 (2020), pp. 231-243 (cit. on p. 2).

[5] Robert M. Corless. "What can we learn from Bohemian matrices?" In: Maple Transactions 1 (1 July 2021), p. 31 (cit. on pp. 1-3, 6, 9, 10).

[6] Ronald L. Graham, Donald E. Knuth, and Oren Patashnik. Concrete Mathematics: A Foundation for Computer Science. 2nd ed. Reading: Addison-Wesley, 1994 (cit. on p. 2).

[7] Andrew Granville. "Zaphod Beeblebrox's Brain and the Fifty-ninth Row of Pascal's Triangle". In: The American Mathematical Monthly 99.4 (Apr. 1992), p. 318. uRL: https://doi.org/10.2307/ 2324898 (cit. on p. 7).

[8] Doug Hardin and Gilbert Strang. "A thousand points of light”. In: The College Mathematics Journal 21.5 (1990), pp. 406-409 (cit. on pp. 5, 6).

[9] Donald E. Knuth. Bracket notation for the 'coefficient of' operator. appeared in: A Classical Mind, essays in honour of C. A. R. Hoare, 1994. 1994. uRL: https://arxiv.org/abs/math/9402216 (cit. on p. 2). 
[10] Longguang Liao and Zexian Cao. "Directional Scaling Symmetry of High-symmetry Twodimensional Lattices”. In: 4.1 (Aug. 2014). uRL: https://doi.org/10.1038/srep06193 (cit. on pp. 5, 6).

[11] D. Steven Mackey et al. "Numerical methods for palindromic eigenvalue problems: Computing the anti-triangular Schur form”. In: Numerical Linear Algebra with Applications 16.1 (Jan. 2009), pp. 63-86. URL: https://doi.org/10.1002/nla.612 (cit. on p. 9).

[12] Kurt Mahler. "On two extremum properties of polynomials". In: Illinois fournal of Mathematics 7.4 (1963), pp. 681-701 (cit. on p. 10).

[13] George Markowsky. "Misconceptions about the Golden Ratio". In: The College Mathematics fournal 23.1 (Jan. 1992), pp. 2-19. URL: https://doi.org/10.1080/07468342.1992.11973428 (cit. on p. 10).

[14] Ruiwen Shu and Jiuya Wang. The Sharp Erdős-Turán Inequality. 2021. arXiv: 2109.11006 [math.CA] (cit. on p. 11).

[15] Olga Taussky. "Matrices of rational integers". In: Bulletin of the American Mathematical Society 66.5 (1960), pp. 327-345. URL: https://doi.org/10.1090/s0002-9904-1960-10439-9 (cit. on p. 10).

[16] Olga Taussky. "Matrices with trace zero". In: The American Mathematical Monthly 69.1 (1962), pp. 40-42 (cit. on p. 10).

[17] Olga Taussky and John Todd. "Systems of Equations, Matrices and Determinants". In: Mathematics Magazine 26.1 (1952), pp. 9-20. ISSN: 0025570X, 19300980. uRL: http://www.jstor.org/ stable/3029837 (cit. on p. 10).

[18] Wikipedia contributors. Lucas's theorem - Wikipedia, The Free Encyclopedia. https://en. wikipedia.org/w/index.php?title=Lucas's_theorem\&oldid=963403394. [Online; accessed 22-May-2021]. 2020 (cit. on p. 7).

[19] Wikipedia contributors. Pingala - Wikipedia, The Free Encyclopedia. https://en.wikipedia. org/w/index.php?title=Pingala\&oldid=1048646202. [Online; accessed 31-October-2021]. 2021 (cit. on p. 10).

\section{A Shining "A Thousand Points of Light" on the error}

The maximum value of $f(k, m)=\left(\begin{array}{c}m-k \\ k\end{array}\right)$ taken as a continuous function of $k$ occurs for $k=k^{*}=$ $m / 2-3 / 10-\sqrt{5 m^{2}+10 m+9} / 10$, which makes $F(m)=\left(\begin{array}{c}m-k^{*} \\ k^{*}\end{array}\right)$ a continuous function of $m$. But the actual integer maximum takes place either at the integer $f\left(\left\lfloor k^{*}\right\rfloor, m\right)$ or at $f\left(\left\lfloor k^{*}\right\rfloor+1, m\right)$; how can we account for this difference? By multivariate calculus, $f(k, m)=f\left(k^{*}, m\right)+\frac{\partial f}{\partial k} f\left(k^{*}, m\right)\left(k-k^{*}\right)+O\left(k-k^{*}\right)^{2}$, thinking of $k$ first as a continuous variable, and then realizing we can substitue an integer in for $k$ afterwards. [NB: some mathematicians hate $O(x)^{2}$ and insist on $O\left(x^{2}\right)$; a short proof shows that they are the same.] So the difference between the actual integer maximum then will be in the asymptotic limit proportional to $k^{*}-\left\lfloor k^{*}\right\rfloor$. For large $m$, we have

$$
k^{*}=\frac{2}{5+\sqrt{5}} m-\frac{3}{10}-\frac{\sqrt{5}}{10}+O\left(\frac{1}{m}\right)
$$

and even for $m$ as small as 1 the omitted terms are less than $1 / 2$. That means we are trying to understand $k^{*}-\left\lfloor k^{*}\right\rfloor$ for large $m$. If it weren't for the $2 /(5+\sqrt{5})$ this would be periodic with period 1 ; as it is, it has period $T=(5+\sqrt{5}) / 2 \approx 3.618$. This means that it can be expanded in a Fourier series with frequencies $2 \pi \ell / T$ for $\ell=1,2, \ldots$. The qualitative agreement of Figures $1(\mathrm{a})$ and $1(\mathrm{~b})$ is so remarkable that I am confident that computing the partial derivative above would explain the vertical scale as well; I have not done this and I invite you to complete this analysis. 